\title{
Postpartum Aortic Dissection and Pulmonary Embolism in a Patient with Marfan's Syndrome: A Rare and Difficult Clinical Scenario
}

\author{
Sheeza Imtiaz*, Muhammad Kashif Shazlee, Kamran Hameed \\ Department of Radiology, Dr. Ziauddin University Hospital, Karachi, Pakistan
}

Copyright $\bigcirc 2018$ by authors, all rights reserved. Authors agree that this article remains permanently open access under the terms of the Creative Commons Attribution License 4.0 International License

\begin{abstract}
Aortic dissection has an estimated incidence of 2.9 per 100,000 person years in general population. In pregnancy, acute type A aortic dissection has an overall incidence of 0.4 cases per 100,000 person years. In $14-50 \%$ of the cases it is associated with Marfan's syndrome. It is a complex clinical scenario, difficult to diagnose and difficult to treat, considering the survival of both mother and fetus. Pregnant women associated with aortic dissection are a special group of patients and a team with multidisciplinary expertise would be important to ensure the best clinical decisions for its treatment. Here in, we present a case of Stanford type "A" aortic dissection along with pulmonary embolism diagnosed in postpartum period in a young patient with Marfan's syndrome.
\end{abstract}

Keywords Pregnancy, Postpartum, Marfan's Syndrome, Aortic Dissection, Pulmonary Embolism

\section{Introduction}

Aortic dissection is a rare disease with estimated incidence of 2.9 per 100,000 person years in general population. [1, 2] and 2,000 patients per year in the United States, of whom $5.3 \%$ are women of reproductive age. Of these, $14-50 \%$ of the cases are associated with Marfan's syndrome and $34-71 \%$ with hypertension. [3, 4] It is a complex clinical scenario, difficult to diagnose and difficult to treat, considering the survival of both mother and fetus. Here in, we present a case of Stanford type A aortic dissection along with pulmonary embolism in a young patient with Marfan's syndrome during postpartum period.

\section{Case Report}

A 33 year old woman (Para 2) known case of Marfan’s
Syndrome was shifted to the emergency department with acute onset of severe chest pain and shortness of breath. The patient was hemodynamically unstable with tachycardia, tachypnea and raised blood pressures. She also had bilateral pedal edema. The patient was in her early postpartum period and gave birth to a full term baby through Caesarian section 20 days back. There were no complications during pregnancy and in the post operative period.

Patient was shifted to the ultrasound department for Doppler examination of both lower limbs for venous insufficiency and was found to have deep venous thrombosis extending up to sapheno-femoral junction bilaterally. Suspecting Pulmonary embolism, patient was immediately shifted for CT Pulmonary angiography that revealed dilated main pulmonary trunk, right and left main pulmonary arteries representing pulmonary arterial hypertension. [Figure.1] Furthermore, there was extensive bilateral pulmonary embolism with presence of thrombus in both main pulmonary arteries and their principle and segmental peripheral branches resulting in multiple patches of pulmonary infarcts and consolidation in both lungs. [Figure 2] Mild bilateral pleural and pericardial effusion was also present. There was also extravasation of contrast in to supra-diapghragmatic IVC and hepatic veins representing associated tricuspid regurgitation.

Additionally, CT Scan revealed dilatation of arch, ascending and descending thoracic aorta with intimal flap extending from aortic root and involving ascending, arch, descending thoracic and abdominal aorta up to the ostium of celiac trunk at D12 level. [Figure3a, 3b] Superiorly, the intimal flap was also extending into proximal part of brachiocephalic trunk, left common carotid and left subclavian arteries. Findings were representing Stanford Type A Aortic dissection. Patient was in severe distress and unfortunately, as soon as the patient was shifted to intensive care unit, she collapsed and expired. 


\section{Discussion}

Aortic dissection during pregnancy and postpartum period is a life threatening condition with high risk of morbidity and mortality for both mother and fetus. The predisposing factors for aortic dissection include hypertension, vasculitis, aneurysm, atherosclerosis, aortic valve replacement, co-arctation of aorta, trauma, cardiac catheterization and collagen vascular diseases like Marfan's syndrome, Ehler'sDanlos syndrome. [5] This case is unique as there are only few case reports of Type A aortic dissection during postpartum period as type B are more prevalent. Further, patient had associated extensive pulmonary embolism which was not reported previously. Up to authors' knowledge, this is the first case report of aortic dissection in a patient with marfan's syndrome during postpartum period in Pakistan. Unfortunately, our patient had a bad outcome and died due to late presentation.

Marfan's syndrome is an autosomal dominant connective tissue disorder and clinical characteristics include symptoms of the cardiovascular, skeletal, and ocular systems. Cardiovascular complications are the main cause of morbidity and mortality in patients with Marfan's syndrome. [6] According to the Stanford classification system, type A dissection involves the ascending aorta with or without involvement of the descending portion, whereas a type B dissection involves the descending aorta alone. [7] Marfan's syndrome is typically related with proximal aortic dilatation; consequently, type A dissections are much more frequent than type B. [8] In a study conducted by Immer et al., [9] 78.9\% of the cases were acute type A dissections and $21.1 \%$ were type B dissections. Results of another study by Konishi and colleague [10] demonstrated $89 \%$ and $11 \%$ of type $\mathrm{A}$ and type $\mathrm{B}$ dissections, respectively. A large study conducted over a period of 27 years described that only $15 \%$ of patients presented with an initial clinical diagnosis of aortic dissection. [11]

Pregnancy is associated with approximately half of the aortic dissection observed in women under 45 years of age. Although the exact patho-physiology between pregnancy and aortic dissection are still unknown, it has been hypothesized that hormonal and physiologic changes of pregnancy may provoke the development of aortic dissection. Due to the hyperdynamic state and effect of hormones on vasculature it usually occurs in the third trimester of pregnancy and during postpartum period. [9] Literature review from 1988 to 2012 yielded that postpartum aortic dissection usually occurred between Day 1 and 42 after delivery and Pregnancy alone without underlying contributing risk factors and Marfan syndrome were the two main risk factors accounting for $44.4 \%$ and $40.7 \%$ of cases, respectively. [12]

A recent study revealed an overall incidence of 0.4 cases per 100,000 person-years of acute type A aortic dissection in pregnancy and a pre-hospital mortality rate of $53 \%$. [13]The mortality rate for untreated proximal aortic dissections increases by $1-3 \%$ per hour after presentation and is roughly $25 \%$ during the first 24 hours and up to $70 \%$ at 1 week and $80 \%$ at 2 weeks. [14] An aortic diameter $\geq 4.0 \mathrm{~cm}$ and a steady increase in the aortic root dimension during pregnancy are indicators of aortic risk in pregnancy. [15] Meijboom et al. [16] reported that an aortic root diameter of $45 \mathrm{~mm}$ in women with Marfan syndrome during pregnancy seems to be relatively safe.

Patients usually presents with symptoms of sudden onset of severe chest pain, vomiting, shortness of breath and even pericardial temponade. Patients may also present with bronchospasm due to vagal nerve stimulation. Our patient presented with severe shortness of breath and haemodynamic collapse with bilateral leg edema due to associated venous thrombosis. Therefore, it is necessary for emergency physicians to have skills to deal patients in cardiopulmonary arrest and to appropriately diagnose the underlying pathology.

The gold standard test for diagnosing aortic dissection is transesophageal echocardiography (TEE) but it is unlikely to be available in emergency settings. International Registry of Acute Aortic Dissections (IRAD) confirmed sensitivity of TEE to be $99 \%$ with specificity, positive and negative predictive values of $89 \%, 89 \%$ and $99 \%$ respectively. [17] Pericardial effusion is a specific indicator of proximal extension of Type A dissection with imminent rupture and should be evaluated through ultrasound in critically ill pregnant patients in acute dissections. Contrast enhanced CT chest and CT angiography are the preferred modality of choice in emergency department, hospitalized and in serial evaluation of ambulatory patients. It has a sensitivity and specificity of about 95-98\%.[18] MRI is also extremely accurate, noninvasive imaging modality, with a sensitivity of $95 \%$ to $98 \%$ and specificity of $94 \%$ to $98 \%$ for detecting aortic dissection. [19]

The management of patients with marfan's syndrome ideally should be started prior to conception. The women and the family should be informed about the potential risk factors and expected complications. Women should undergo careful examination of all cardiovascular abnormalities like diameter of aortic root, ascending, descending aorta, valvular and left ventricular function. There is $50 \%$ risk of transfer of marfan's syndrome from mother to fetus. Prenatal genetic testing and fetal echocardiography should be done.

Treatment guidelines for aortic dissection depend upon the type of aortic dissection and also on the duration of pregnancy. Stanford type A dissections are generally managed surgically while type B dissections are managed medically. While there are no well-known practice guidelines, it has been recommended that before 28 weeks of gestation, aortic repair should proceed without cesarean section and after 32 weeks gestation, emergency cesarean section precede aortic repair. Between 28 and 32 weeks, the risks and benefits of early delivery should be 
considered. [20] IV nitroprusside, if presenting with dangerously elevated pressures, and a $\beta$-blocker should be started upon initial diagnosis. Blood pressure control is one of the major actions that can be taken to avoid the development of aortic dissections in pregnancy, and to prevent further worsening of them if they have already developed. Cardiac surgery during pregnancy is associated with increased risk to both mother and fetus with a reported maternal mortality of $8.7 \%$ and increase intrauterine deaths. Transthoracic echocardiography should be performed every 4-6 weeks in aortic root diameter of $>40 \mathrm{~mm}$, progressive dilatation and in every trimester with normal sized aorta.

\section{Conclusions}

There is an eight fold increase risk of aortic dissection in pregnant females with marfan's syndrome. Lack of knowledge and inappropriate diagnosis leads to higher rates of maternal and fetal mortality. Emergency

physicians and clinicians must maintain a high index of suspicion for aortic dissection in any pregnant female who presents with acute onset chest pain. Early recognition, in addition to successful co-ordination of care between the emergency physician, obstetrician, and cardiothoracic/vascular surgeon is necessary for a successful outcome. During pregnancy, the maternal and fetal risks should be assessed by the obstetrician, cardiologist and genetic specialist. Patients should be councelled about the risk factors and complications associated with it. Event free pregnancy cannot be guaranteed even in normal aortic diameter and complications can occur any time during pregnancy and in postpartum period. Patients should be told of the importance of close follow up and be educated of the symptoms of aortic dissection and an emergent need of immediate care.

\section{Figure Legends}

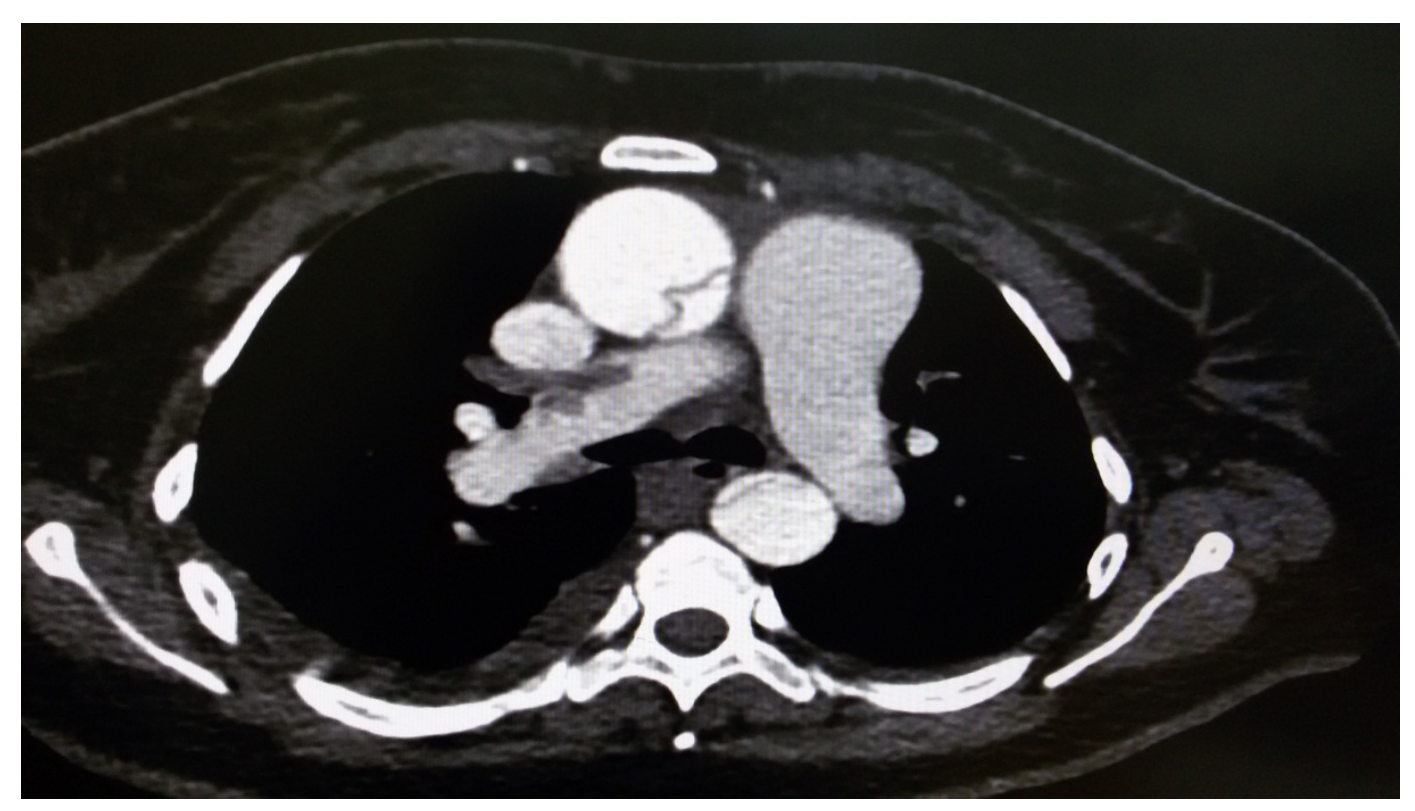

Figure1. CT scan Pulmonary angiography axial scan showing dilatation of main pulmonary trunk and its right and left branches representing pulmonary arterial hypertension with pulmonary embolism due to a hypodense thrombus in right main pulmonary artery. Aortic dissection also seen involving ascending and descending thoracic aorta 


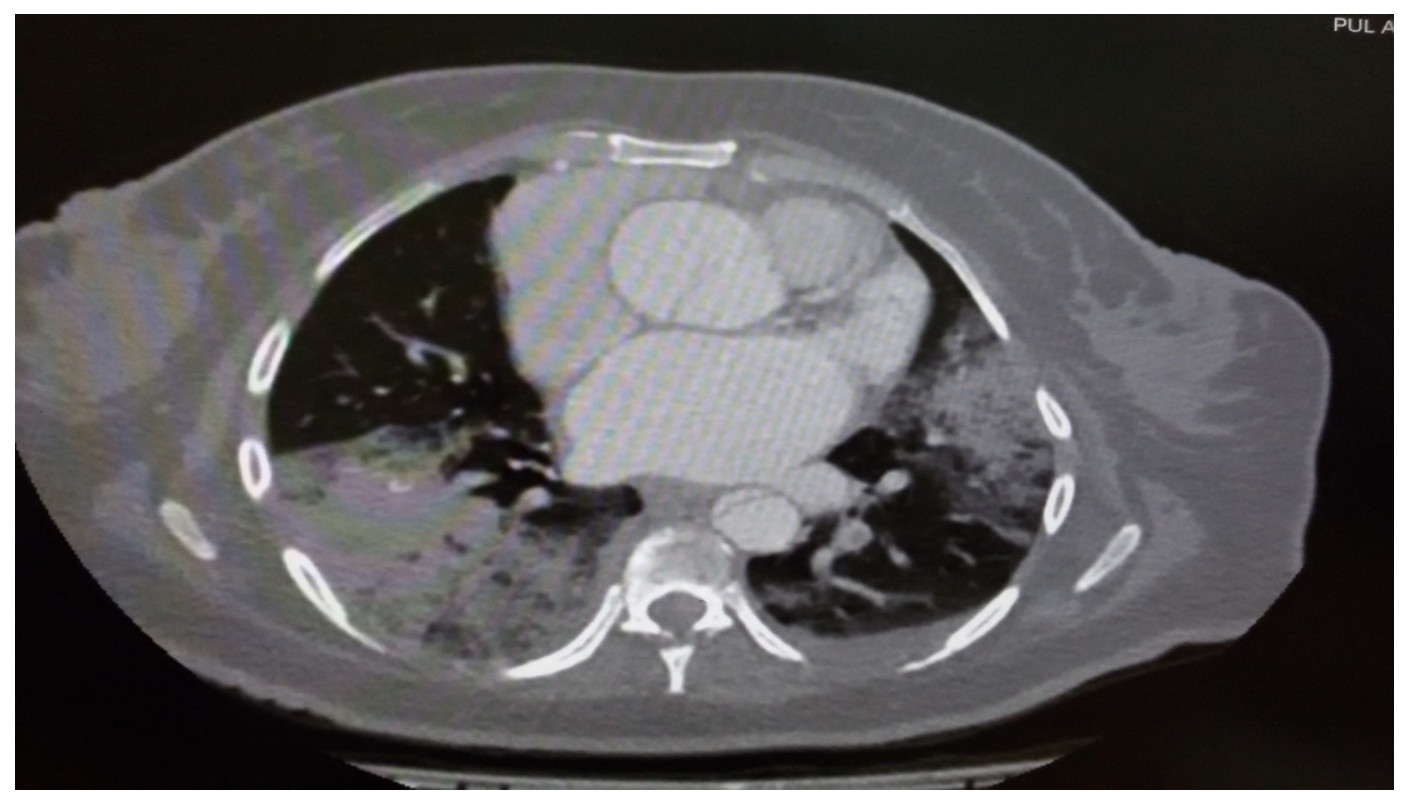

Figure 2. CT scan Pulmonary angiography axial scan in lung window setting showing patch of pulmonary infarct / consolidation involving both lungs with mild bilateral pleural effusion

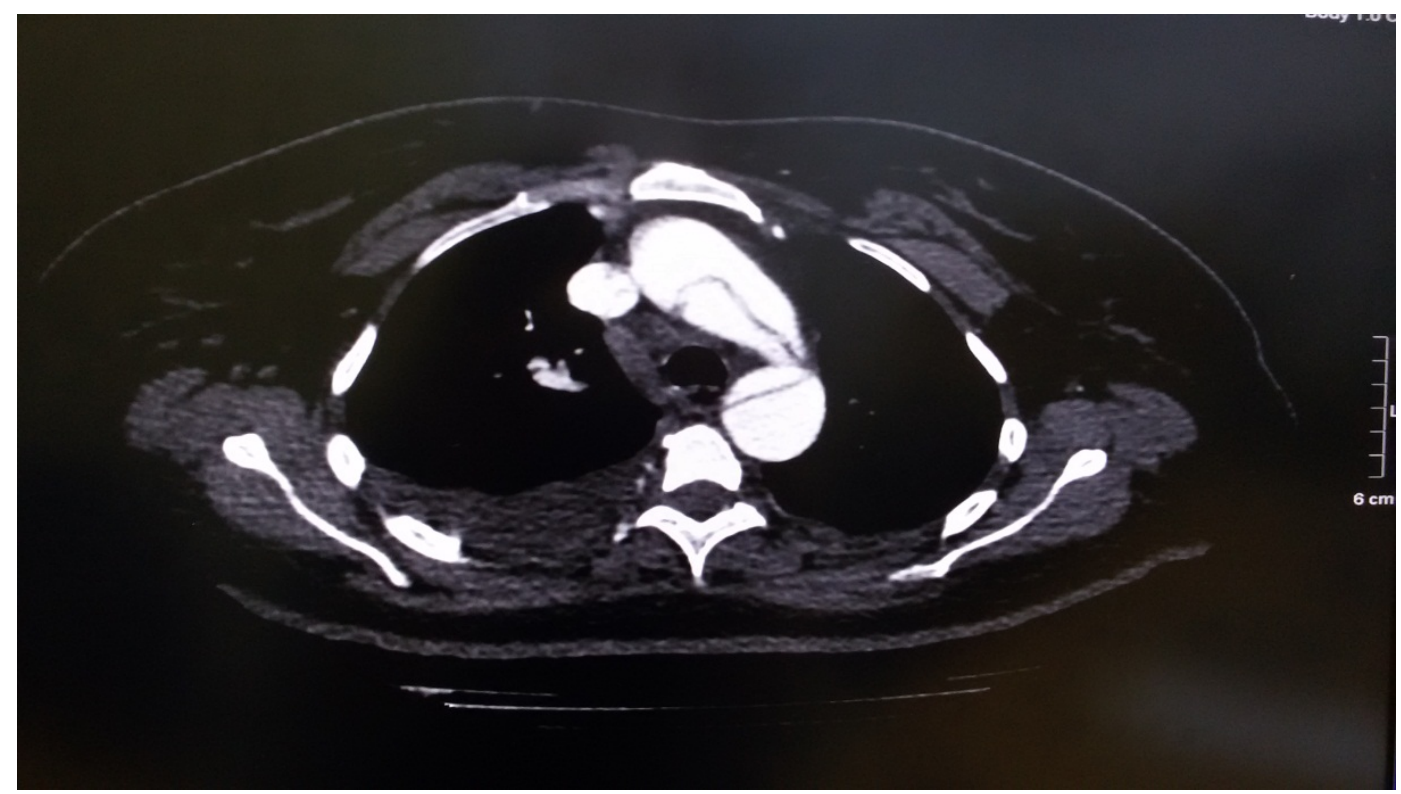

Figure 3a. CT scan Pulmonary angiography axial scan showing intimal flap involving ascending, arch and descending thoracic aorta dividing into true and false lumen representing Stanford type A aortic dissection. Mild bilateral pleural effusion also seen 


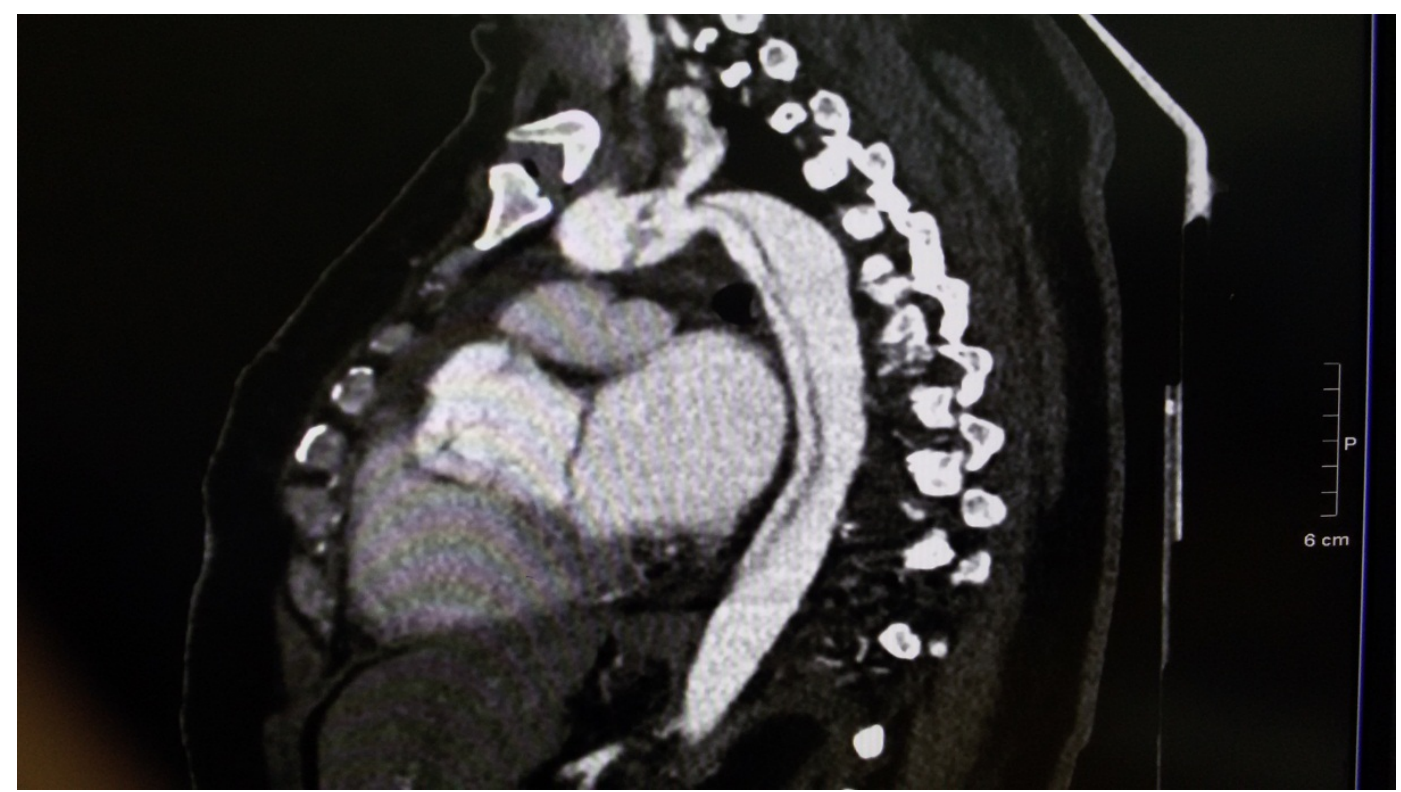

Figure 3b. CT scan Pulmonary angiography sagittal scan showing intimal flap involving aortic root, ascending, arch and descending thoracic aorta dividing into true and false lumen representing Stanford type A aortic dissection

\section{REFERENCES}

[1] Bickerstaff LK, Pairolero PC, Hollier LH, Melton LJ, Van Peenen HJ, Cherry KJ, Joyce JW. et al. Thoracic aortic aneurysms: a population-based study. Surgery. 1982; 92(6):1103-1108.

[2] Clouse WD, Hallett JW Jr, Schaff HV, Spittell PC, Rowland CM, Ilstrup DM, Melton LJ 3rd. Acute aortic dissection: population-based incidence compared with degenerative aortic aneurysm rupture. Mayo Clin Proc.2004;79(2):176-180. doi: 10.4065/79.2.176.

[3] Januzzi JL, Isselbacher EM, Fattori R, Cooper JV, Smith DE, Fang J, et al. Characterizing the young patient with aortic dissection: results from the International Registry of Aortic Dissection (IRAD)J Am CollCardiol.2004;43:665669.

[4] Oskoui R, Lindsay J., Jr Aortic dissection in women $<40$ years of age and the unimportance of pregnancy. Am J Cardiol.1994;73:821-823.

[5] Elkayam U, Ostrzega E, Shotan A, Mehra A. Cardiovascular problems in pregnant women with the Marfan syndrome. Ann Intern Med.1995;123(2):117-122. doi: 10.7326/0003-4819-123-2-199507150-00007.

[6] Judge DP, Diez HC. Marfan's syndrome. Lancet. 2005;366:1965-1976.

[7] Daily PO, Trueblood HW, Stinson EB, Wuerflein RD, Shumway NE. Management of acute aortic dissections. Ann Thorac Surg.1970;10:237-247.

[8] Mimoun L, Detaint D, Hamroun D.Dissection in Marfan syndrome: the importance of the descending aorta. Eur Heart J. 2011;32:443-449.
[9] Immer FF, Bansi AG, Immer-Bansi AS, et al. Aortic dissection in pregnancy: analysis of risk factors and outcome. Ann Thorac Surg. 2003; 76: 309-314 doi: 10.1016/S0003-4975(03)00169-3.

[10] Konishi Y, Tatsuta N, Kumada K. Dissecting aneurysm during pregnancy and the puerperium. JpnCirc J.1980;44:726-733.

[11] Mészáros, J. Mórocz, J. Szlávi et al. Epidemiology and clinicopathology of aortic dissection: a population-based longitudinal study over 27 years. Chest. 2000;117(5):12711278 ,

[12] Shi-min yuan. Postpartum aortic dissection. Taiwanese journal of obstetric nadgynaecology. 2013;52(3):318-322.

[13] M. Thalmann, G. H. Sodeck, H. Domanovits et al. Acute type A aortic dissection and pregnancy: a population-based study. European Journal of Cardio-thoracic Surgery. 2011;39(6):159-163.

[14] M. P. I. Pitt and R. S. Bonser. The natural history of thoracic aortic aneurysm disease: an overview. Journal of Cardiac Surgery. 1997;12(2):270-278.

[15] Task Force on the Management of Cardiovascular Diseases during Pregnancy of the European Society of Cardiology. Expert consensus document on management of cardiovascular diseases during pregnancy. Eur Heart J. 2003; $4: 761-781$

[16] Meijboom LJ, Timmermans J, Zwinderman AH, Engelfriet PM, Mulder BJ. Aortic root growth in men and women with the Marfan's syndrome. Am J Cardiol. 2005;96:1441-1444.

[17] A.G.Moore, K.A. Eagle, D.Bruckman et al. Choice of computed tomography, transesophageal echocardiography, magnetic resonance imaging, and aortography in acute aortic dissection: International Registry of Acute Aortic Dissection (IRAD). Am J Cardiol. 2002;89:1235-1238.

[18] M.A. McMahon, C.A. Squirrell. Multidetector CT aortic 
dissection: a pictorial review. Radiographics. review and meta-analysis. Arch Intern Med. 2010;30:445-446. 2006; $166: 1350-1356$

[19] T. Shiga, Z. WAjima, C.C. Apfel, T. Inoue, Y. Ohe. Diagnostic accuracy of tarnsesophageal echocardiography, helical computed tomography, and magnetic resonance imaging for suspected thoracic aortic dissection: systematic

[20] C. J. Zeegbregts, M. A. Schepens, T. M. Hameeteman, W. J. Morshuis, and A. B. de la Rivière, "Acute aortic dissection complicating pregnancy. Ann Thorac Surg. 1997;64:13451348. 